\title{
Clinical care standards: appropriate care everywhere - acute coronary syndromes as an example
}

\author{
Derek P Chew ${ }^{1,2}$, Robert Herkes ${ }^{2}$, Meredith A Page ${ }^{2}$
}

A ustralian practice data from clinical audits and other research have clearly shown that dissemination of high quality clinical practice guidelines is not enough to ensure everyone with a particular clinical condition receives appropriate care. ${ }^{1-3}$ Even for clinical conditions with established networks of care, such as acute coronary syndromes (ACS), variations in care persist, not all of which can be explained by patient needs or preferences. In this article, we explore the features and underlying principles of clinical care standards (looking at the example of ACS) and the improvements in delivery of appropriate care that a clinical care standard may help to make.

The National Health Reform Agreement signed by the Council of Australian Governments in 2011 (http://www.federalfinancial relations.gov.au/content/npa/health/_archive/national-agreement. pdf) determined that clinical standards would be developed by the Australian Commission on Safety and Quality in Health Care (the Commission) to help ensure appropriateness of care for specific clinical conditions nationally. Clinical care standards are small sets of concise recommendations (quality statements) that focus on specific gaps in evidence-based practice for a clinical condition. Their aim is to drive delivery of appropriate care, reduce unwarranted variation and to promote shared decision making between patients, carers and clinicians.

\section{What's different about a clinical care standard?}

Unlike a clinical practice guideline, a clinical care standard is not a comprehensive management guide. Instead, it targets areas of care where both divergence from evidence and the opportunity for improvement are greatest. In collaboration with a working group of clinicians, researchers, health care organisations and consumers, the Commission uses practice data to identify key areas for improvement, which become the focus of each quality statement.

Clinical care standards are developed using a process designed to optimise the uptake and reach of the care they describe. First, each topic requires the agreement of representatives of state and territory health departments. Second, before public consultation, the draft standard is considered by representatives from private and public health sectors. Third, each draft is released for broad public consultation, with feedback sought from all levels of the health system as well as from key organisations. Finally, before it is released, the standard is submitted to the Australian Health Ministers Advisory Council and then to Australian Health Ministers. This highly collaborative and consultative method of development not only assists in ensuring the relevance of the standard to the health care system but builds engagement of both clinical and policy decision makers at multiple levels within the system. In prompting review of existing initiatives, the standard acts as a focus for integrated whole-of-system efforts to improve the quality of care.
Summary

- Audit data show that despite clinical practice guidelines, some patients miss out on evidence-based care, which is not explained by individual needs or preferences.

- Clinical care standards are small sets of concise recommendations that focus on known gaps in evidencebased care for a particular clinical condition. They aim to ensure that all patients with the same clinical condition are offered appropriate care, regardless of their location.

- Clinical care standards are nationally agreed standards and are developed to maximise engagement of consumers, clinicians, health services, and state and territory health departments and agencies. They complement clinical practice guidelines and other initiatives for improving quality of health care.

- As an example, the Acute Coronary Syndromes (ACS) Clinical Care Standard is a significant step forward in supporting clinicians and health services to realise all of the gains promised by the ACS evidence base.

- The ACS Clinical Care Standard focuses on the areas of care that are known to be most associated with variation in outcome. It supports patient involvement in critical decisions that affect their care, refocuses clinicians on the priority areas of ensuring appropriate ACS care, and informs health services about the systems required to deliver evidencebased care.

Putting patients at the centre of care is an integral part of clinical care standards and is ensured in several ways:

- the quality statements are written from the patient's perspective, describing the care that patients can expect to be offered by the health care system;

- a consumer fact sheet accompanies each standard describing in lay terms what the quality statement means; and

- the standards align understanding and expectations of patients, clinicians and health services about what good care is, how it should be delivered, and what needs to be put in place to ensure it happens.

Indicators are an important part of implementation support for each clinical care standard. Each standard has a set of indicators for local use by health services to monitor adherence and to identify areas for improvement. The National Stroke Foundation's 2015 national audit recently showed how the process indicators from a standard can be used to track performance over time. ${ }^{2}$ The indicators can also be used by health services to show they are providing high quality evidence-based care, a requirement of the National Safety and Quality Health Service Standards. ${ }^{4}$ The indicators are not mandatory, as some services may have other measures in place to demonstrate they are delivering the care described within a standard. 
1 Acute Coronary Syndromes Clinical Care Standard ${ }^{5}$

1. A patient presenting with acute chest pain or other symptoms suggestive of an acute coronary syndrome receives care guided by a documented chest pain assessment pathway.

2. A patient with acute chest pain or other symptoms suggestive of an acute coronary syndrome receives a 12-lead electrocardiogram (ECG) and the results are analysed by a clinician experienced in interpreting an ECG within 10 minutes of the first emergency clinical contact.

3. A patient with an acute ST-segment-elevation myocardial infarction (STEMI), for whom emergency reperfusion is clinically appropriate, is offered timely percutaneous coronary intervention $(\mathrm{PCl})$ or fibrinolysis in accordance with the time frames recommended in the current National Heart Foundation of Australia/Cardiac Society of Australia and New Zealand Guidelines for the management of acute coronary syndromes. In general, primary $\mathrm{PCl}$ is recommended if the time from first medical contact to balloon inflation is anticipated to be less than 90 minutes, otherwise the patient is offered fibrinolysis.

4. A patient with a non-ST-segment-elevation acute coronary syndrome (NSTEACS) is managed based on a documented, evidence-based assessment of their risk of an adverse event.

5. The role of coronary angiography with a view to timely and appropriate coronary revascularisation is discussed with a patient with a non-ST-segment-elevation acute coronary syndrome (NSTEACS) who is assessed to be at intermediate or high risk of an adverse cardiac event.

6. Before a patient with an acute coronary syndrome leaves the hospital, they are involved in the development of an individualised care plan. This plan identifies the lifestyle modifications and medicines needed to manage their risk factors, addresses their psychosocial needs and includes a referral to an appropriate cardiac rehabilitation or another secondary prevention program. This plan is provided to the patient and their general practitioner or ongoing clinical provider within 48 hours of discharge.

Australia is the second country to develop national clinical standards. In 2009, the National Institute for Health and Care Excellence (NICE) established a program to develop quality standards across health care, public health and social care, for use in England. As of December 2015, NICE had developed over 100 quality standards. The Commission's Clinical Care Standard program began in 2013. Standards on ACS, antimicrobial stewardship, acute stroke, delirium and hip fracture care have been released. Standards for osteoarthritis of the knee and heavy menstrual bleeding are under development.

\section{Improving care for patients with acute coronary syndromes}

The ACS Clinical Care Standard was developed to address substantial heterogeneity in the use of treatments known to reduce risk of cardiac events, which could not be explained by clinical complexity or patient needs. ${ }^{1}$ Research into the sources of this variation offers insights into potential targets for improving ACS care throughout Australia, and was used to inform the quality statements of the Standard (Box 1).

\section{Practice-related factors}

Translation of knowledge into practice has been identified as a major practice-related issue affecting quality of ACS care. ${ }^{6}$ In a 2009 survey, Australian clinicians reported high levels of agreement with, and adherence to, the existing (2006) ACS guidelines. However, these views correlated poorly with observed practice, with often striking overestimation by clinicians of their use of treatments recommended in the guidelines. ${ }^{6}$

Clinician misperception about patient risk of recurrent cardiovascular events and uncertainty about applying evidence for patients with complex clinical needs appear to be contributors to the variation. Cardiovascular risk stratification is a critical decision point in the appropriate provision of cardiac care. Compared with assessment using validated cardiovascular risk tools, clinical intuition more frequently overestimates the risk for low risk patients and underestimates that for high risk patients. ${ }^{7,8}$ Underappreciation of cardiovascular risk is worse for females with myocardial infarction (MI) than for males with MI and is associated with delays in providing invasive management, lower use of evidence-based drugs and an increase in the risk of late mortality.
Even when risk is accurately estimated, challenges remain in applying the ACS evidence base among patients with complex clinical needs. Australian data show that patients with comorbidities are less likely to receive evidence-based treatments than patients at the same level of cardiovascular risk without comorbidities. ${ }^{10}$ Age and clinical comorbidities, such as prior cerebrovascular disease, lung disease and impaired renal function, elevate the risk of adverse events from invasive management (eg, reperfusion) and cardiovascular therapies (eg, antiplatelet drugs). However, the marked divergence in care for these patients suggests that intervention-related risks are not being objectively weighed up against treatment benefits (Box 2). ${ }^{10}$

Risk stratification and discussion of these risks and the benefits of coronary angiography for non-ST-segment-elevation ACS are the focus of quality statements 4 and 5 of the Standard (Box 1 ).

\section{System factors}

There are several important system-related contributors to ACS practice variation. The absence of systematic processes to improve the timely provision of reperfusion in ST-segment-elevation MI (STEMI) results in longer "door to balloon time" and is associated with increased rates of mortality and recurrent MI. ${ }^{11,12}$ In a survey of 35 hospitals examining implementation strategies for reducing

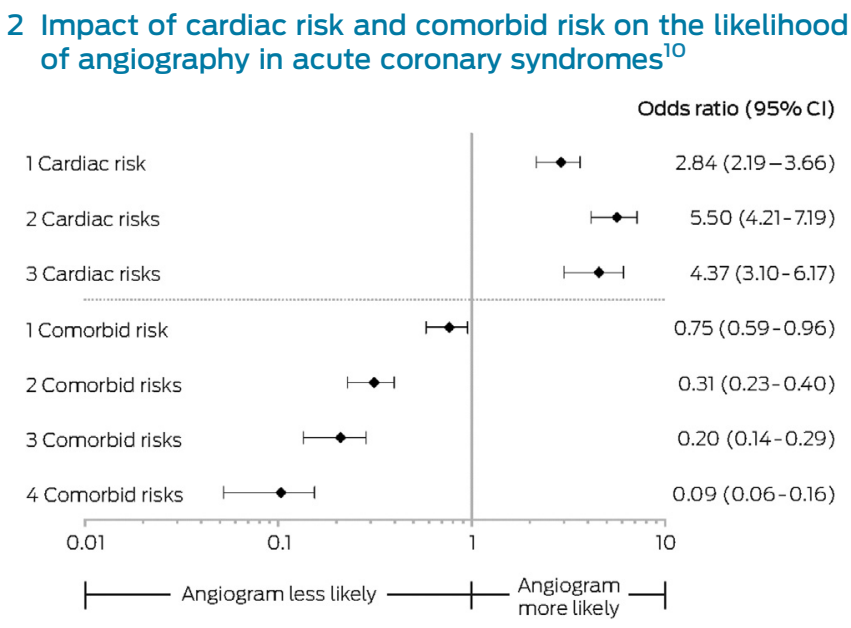

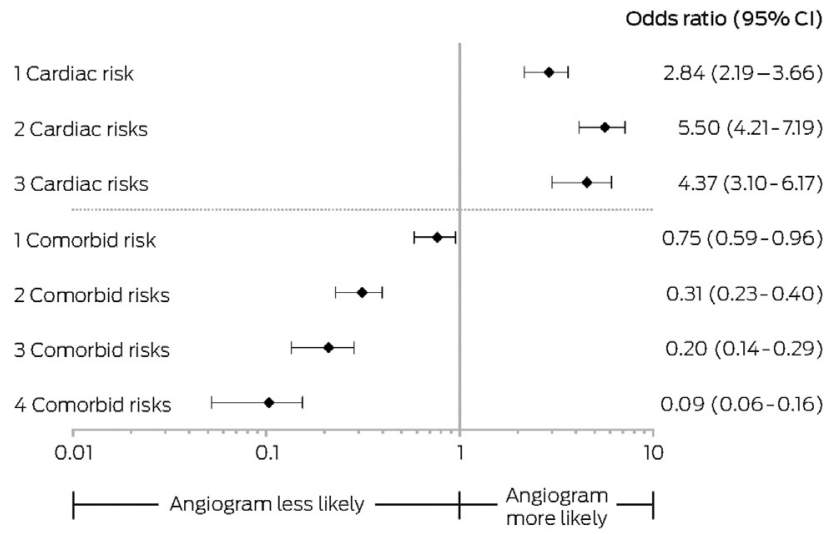


time to reperfusion, those with at least one strategy delivered reperfusion on average 21 minutes faster than those without any strategy, and this was associated with a reduced rate of 12-month death or MI $(12.8 \%$ v $22.1 \%$, respectively; $P=0.006){ }^{12}$

The need for systematic processes to improve the timeliness and accuracy of diagnosis is highlighted in quality statements 1 and 2 of the Standard; quality statement 3 focuses on systems to support the timely provision of reperfusion (Box 1 ).

The vastness of Australia's geography and the nation's cultural diversity are both possible contributors to ACS practice variation and to patient outcomes. Mortality and complication rates due to MI are higher in rural and regional areas than in metropolitan areas; ${ }^{1,13}$ lack of access to clinical expertise and to timely invasive management for non-ST-segment-elevation ACS may contribute to variation in these areas of care. ${ }^{14}$ Variation in prescription of secondary prevention medications and lifestyle modifications, and in the engagement of patients to adhere to them, are likely to be particularly challenging gaps to address in the diverse demographic profile of modern Australia, yet they are important targets for well-designed interventions.

Quality statement 6 of the Standard focuses on use of an individualised care plan to improve the transition of care, including the ongoing need for secondary prevention measures (Box 1).

\section{Impact of reducing variation}

Attempts to estimate the impact of quality initiatives suggest there are substantial gains associated with making system-wide improvements to ACS care. ${ }^{15}$ Several observations are worth highlighting:

- The gains to be had from rectifying omissions of care (eg, missed opportunities in timely reperfusion) far exceed the benefits to be gained from choices between therapies (eg, immediate fibrinolysis versus transfer for percutaneous coronary intervention in STEMI).

- Improving the application of secondary prevention therapies is likely to have a much greater impact on late clinical outcomes than a focus on invasive management.

- The incremental benefit of new treatments and strategies applied to patients who are already optimally treated is relatively small and is dwarfed by the gains to be had for all by small improvements in the delivery and reach of evidencebased care across the whole system. ${ }^{11,12}$

Economic evaluation of strategies to improve the quality of ACS care in Australia are limited. Yet the fact that half of all cardiovascular events occur in patients with prior documented coronary artery disease, and that the estimated yearly costs of MI to the Australian community is $\$ 1.14$ billion dollars, suggests that even modest systems improvements in ACS care are likely to be highly efficient. $^{16}$
Efforts to redesign the provision of care in various parts of Australia have been promising, showing meaningful improvements in patient outcomes. For example, several health networks have sought to move the identification of STEMI into the ambulance, through the transmission of initial echocardiographic data to the emergency department, ensuring patients are brought to hospitals with appropriate clinical teams and facilities, as well as shortening the time to response when these teams are on remote call. ${ }^{17,18}$

Similarly, clinical networks that provide remote clinical support combined with access to metropolitan-based invasive services have been shown to reduce heterogeneity in clinical care and improve short term outcomes for patients residing in rural areas. ${ }^{14}$

Complementary to these initiatives, approaches to secondary prevention that embrace the individuality of patient needs and tailor education and care to these have shown significant promise in improving adherence, as well as the achievement of risk factor targets known to lower overall risk of future cardiovascular events. ${ }^{19,20}$

The uptake of the ACS Clinical Care Standard is not yet known, and neither is that of the UK's corresponding quality standard, also released in 2014. However, substantial improvements in the care and survival for older people with hip fracture in England have been attributed to initiatives underpinned by a national clinical standard, including a national registry to support audit and feedback, and a financial incentives program. ${ }^{21}$ In December 2014 NICE reported on the uptake of 28 published quality standards. While data were limited, uptake of care could be tracked over time for many quality standards using selected process indicators. ${ }^{22}$

\section{What the ACS Clinical Care Standard adds}

The ACS Clinical Care Standard is a significant step forward in supporting clinicians and health services to realise all of the gains promised by the ACS evidence base. ${ }^{23}$ It focuses on the areas of care that are known to be most associated with variation in outcome. It supports patient involvement in critical decisions that affect their care, refocuses clinicians on the priority areas of ensuring appropriate ACS care, and informs health services about the systems required to deliver evidence-based care. The revised ACS clinical practice guidelines released in August 2016 remain consistent with the ACS Clinical Care Standard and specifically support its use for quality improvement. ${ }^{24}$

Ensuring effective and equitable care of patients with ACS across Australia is complex. For the first time, a common vision for care of patients with ACS provides an opportunity for alignment and coordination of local, state and national efforts to improve consistency of care. In doing so, the ACS Clinical Care Standard may just represent the keystone for evidence translation in Australian ACS care.

Competing interests: No relevant disclosures.

Provenance: Commissioned; externally peer reviewed.

(C) 2016 AMPCo Pty Ltd. Produced with Elsevier B.V. All rights reserved.
1 Chew DP, French J, Briffa TG, et al. Acute coronary syndrome care across Australia and New Zealand: the SNAPSHOT ACS study. Med J Aust 2013; 199: 185-191. https://www.mja.com.au/journal/2013/199/3/acutecoronary-syndrome-care-across-australia-and-newzealand-snapshot-acs-study

2 National Stroke Foundation. National Stroke Audit Acute Services Report 2015. Melbourne: National Stroke
Foundation, 2015. https://informme.org.au/en/strokedata/Acute-audits (accessed Oct 2016).

3 Australian Commission on Safety and Quality in Health Care. Antimicrobial prescribing practice in Australia: results of the 2014 National Antimicrobial Prescribing Survey. Sydney: ACSQHC, 2015. http://www.safetyandquality.gov.au/wp-content/ uploads/2015/07/Antimicrobial-prescribing-
practice-in-Aust-hospitals-NAPS-2014-Results.pdf (accessed Dec 2015)

4 Australian Commission on Safety and Quality in Health Care. National Safety and Quality Health Service Standards. Sydney: ACSQHC, 2012. http://www. safetyandquality.gov.au/wp-content/uploads/2011/ 09/NSQHS-Standards-Sept-2012.pdf

(accessed June 2015). 
5 Australian Commission on Safety and Quality in Health Care. Acute Coronary Syndromes Clinical Care Standard. Sydney: ACSQHC, 2014. http://www. safetyandquality.gov.au/wp-content/uploads/2014/12/ Acute-Coronary-Syndromes-Clinical-Care-Standard.pdf (accessed Dec 2015).

6 Huynh LT, Chew DPB, Sladek RM, et al. Unperceived treatment gaps in acute coronary syndromes. Int I Clin Pract 2009; 63: 1456-1464.

7 Scott I, Derhy P, O'Kane D, et al. Discordance between level of risk and intensity of evidence-based treatment in patients with acute coronary syndromes. Med $\mathrm{J}$ Aust 2007; 187: 153-159. https://www.mia.com.au/ journal/2007/187/3/discordance-between-level-riskand-intensity-evidence-based-treatment-patients

8 Chew DP, Junbo G, Parsonage W, et al. Perceived risk of ischemic and bleeding events in acute coronary syndromes. Circ Cardiovasc Qual Outcomes 2013; 6: 299-308.

9 Chew DP, Juergens C, French J, et al. An examination of clinical intuition in risk assessment among acute coronary syndromes patients: Observations from a prospective multi-center international observational registry. Int J Cardiol 2014; 171: 209-216.

10 Joynt KE, Huynh L, Amerena JV, et al. Impact of acute and chronic risk factors on use of evidence-based treatments in patients in Australia with acute coronary syndromes. Heart 2009; 95: 1442-1448.

11 Bradley E, Herrin J, Wang Y, et al. Strategies for reducing the door-to-balloon time in acute myocardial infarction N Engl J Med 2006; 355: 2308-2320.

12 Astley CM, Macdougall CJ, Davidson PM, Chew DP. Lost in translation: health resource variability in the achievement of optimal performance and clinical outcome. Circ Cardiovasc Qual Outcomes 2011; 4 : 512-520.

13 Cullen L, French JK, Briffa TG, et al. Availability of highly sensitive troponin assays and acute coronary syndrome care: insights from the SNAPSHOT registry. Med $J$ Aust 2015; 202: 36-39. https://www.mja.com.au/ journal/2015/202/1/availability-highly-sensitivetroponin-assays-and-acute-coronary-syndrome-care

14 Tideman PA, Tirimacco R, Senior DP, et al. Impact of a regionalised clinical cardiac support network on mortality among rural patients with myocardial infarction. Med J Aust 2014; 200: 157-160. https://www. mja.com.au/journal/2014/200/3/impact-regionalisedclinical-cardiac-support-network-mortality-among-rural

15 Chew DP, Huynh LT, Liew D, et al. Potential survival gains in the treatment of myocardial infarction. Heart 2009: 95: 1844-1850.

16 Ionannides-Demos LL, Makarounas-Kirchmann K, Ashton $\mathrm{E}$, et al. Cost of myocardial infarction to the Australian community: a prospective, multicentre survey. Clin Drug Investig 2010; 30: 533-543.

17 Carstensen S, Nelson GCl, Hansen PS, et al. Field triage to primary angioplasty combined with emergency department bypass reduces treatment delays and is associated with improved outcome. Eur Heart J 2007; 28: 2313-2319.

18 Hutchison AW, Malaiapan Y, Cameron JD, Meredith IT. Pre-hospital 12 lead ECG to triage ST elevation myocardial infarction and long term improvements in door to balloon times: The first 1000 patients from the MonAMI project. Heart Lung Circ 2013; 22: 910-916.
19 Redfern J, Briffa T, Ellis E, Freedman SB. Choice of secondary prevention improves risk factors after acute coronary syndrome: 1-year follow-up of the CHOICE (Choice of Health Options In prevention of Cardiovascular Events) randomised controlled trial. Heart 2009; 95: 468-475.

20 Redfern J, Ellis E, Briffa T, Freedman SB. Modular prevention of heart disease following acute coronary syndrome (ACS) [ISRCTN42984084]. BMC Cardiovasc Disord 2006; 6: 26.

21 Neuburger J, Currie C, Wakeman R, et al. The impact of a national clinician-led audit initiative on care and mortality after hip fracture in England: an external evaluation using time trends in non-audit data. Med Care 2015; 53: 686-691.

22 National Institute for Health and Care Excellence. The uptake of NICE quality standards: reporting on quality standards QSI-57. London: NICE, 2014. https://www. nice.org.uk/media/default/Standards-and-indicators/ Quality-standards/qs-uptake-report-dec-14.pdf (accessed Dec 2015).

23 Chew DP, Briffa TG. The Clinical Care Standards in ACS: towards an integrated approach to evidence translation in ACS care. Heart Lung Circ 2015; 24: 213-215.

24 Chew DP, Scott IA, Cullen L, et al. National Heart Foundation of Australia and Cardiac Society of Australia and New Zealand: Australian clinical guidelines for the management of acute coronary syndromes 2016. Med J Aust 2016; 205: 128-133. https://www.mja.com.au/ journal/2016/205/3/national-heart-foundationaustralia-and-cardiac-society-australia-and-newzealand 